NUCLEUS-NUCLEUS COLLISIONS AT 60 TO $200 \mathrm{GeV} /$ NUCLEON: RESULTS FROM THE WA80 EXPERIMENT AT CERN

\title{
WA80 Collaboration
}

F. Plasil, ${ }^{a}$ R. Albrecht, ${ }^{b}$ T. C. Awes, ${ }^{a}$ C. Baktash, ${ }^{a}$ P. Beckmann, ${ }^{c}$ F. Berger, ${ }^{c}$

R. Bock, ${ }^{b}$ G. Claesson, ${ }^{b}$ G. Clewing, ${ }^{c}$ L. Dragon, ${ }^{c}$ A. Eklund, ${ }^{d}$ R. L. Ferguson, ${ }^{a}$

A. Franz, ${ }^{e, f}$ S. Garpman, ${ }^{d}$ R. Giasow, ${ }^{c}$ H. A. Gustafsson, ${ }^{d}$ H. H. Gutbrod, ${ }^{b}$

J. Idh, ${ }^{d}$ P. Jacobs, ${ }^{e}$ K. H. Kampert, ${ }^{c}$ B. W. Kolb, ${ }^{b}$ P. Kristiansson, ${ }^{e}$ I. Y. Lee, ${ }^{a}$

H. Löhner, ${ }^{c}$ I. Lund, ${ }^{b}$ F. E. Obenshain, ${ }^{a, j}$ A. Oskarsson, ${ }^{d}$ I. Otterlund, ${ }^{d}$

T. Peitzmanı, ${ }^{c}$ S. Persson, ${ }^{d}$ A. M. Poskanzer, ${ }^{e}$ M. Purschke, ${ }^{c}$ H. G. Ritter, ${ }^{e}$

S. Saini, ${ }^{a}$ R. Santo, ${ }^{c}$ H. R. Schmidt, ${ }^{b}$ T. Siemiarczuk, ${ }^{b}$ S. P. Sorensen, ${ }^{a, f}$

K. Steffens, ${ }^{c}$ E. Stenlund, ${ }^{d}$ D. Stüken, ${ }^{c}$ M. L. Tincknell, ${ }^{a}$ and G. R. Young ${ }^{a}$

a Oak Ridge National Laboratory, ${ }^{*}$ Oak Rjdge, Tennessee 37831

- Gesellschaft für Schwerionenforschung (GSI), D-6100 Darmstadt, West Germany

c University of Münster, D-4400 Münster, West Germany

University of Lund, S-22362 Lund, Sweden

- Lawrence Berkeley Laboratory, Berkeley, California 94720

$f$ University of Tennessee, Knoxville, Tennessee 37996

Results from ${ }^{16} \mathrm{O}$ - and ${ }^{32} \mathrm{~S}$-induced reactions obtained by the WA80 collaboration at the CERN SPS are presented with reference to global event characteristics such as collision geometry, the degree of nuclear stopping, and the energy density attained. Transverse momentum spectra of neutral pions and of direct photons are also presented. At an accuracy within $15 \%$ limits, all observed photons are accounted for by known hadronic decays.

\section{INTRODUCTION}

Three and one-half years have elapsed since nuclei with ultrarelativistic energies became available at CERN and at BNL. In this paper, we summarize the main points that we have learned from the WA80 experiment as a result of the first round of experiments with ${ }^{16} \mathrm{O}$ and ${ }^{32} \mathrm{~S}$ projectiles having energies up to $200 \mathrm{GeV} /$ nucleon. It is worth noting that all CERN SPS results were obtained by means of a very limited amount of beam time. Only about two weeks of ${ }^{16} \mathrm{O}$ beams and two weeks of ${ }^{32} \mathrm{~S}$ beams have been made available thus far. Nevertheless, a wealth of information on nucleus-nucleus collisions at these extreme energies has been obtained by WA80 and by other collaborations. Perhaps the most satisfying aspect of this venture is the understanding that has been gained concerning the global fea ${ }^{\wedge}$ ures of the reactions. While these have been discussed before, ${ }^{1}$ we present here the main characteristics together with newly analyzed systematic results involving transverse-energy pseudorapidity distributions, ${ }^{2}$ which allow us to focus on a number of subtle effects which were not apparent in the rapidity-integrated data.

Each major CERN! experiment has concentrated on one specific method by which the formation of the quark-gluon plasma (QGP) can be studied. WA80 has emphasized the

* Oak Ridge National Laboratory is operated by Martin Marietta Energy Systems, Inc., under contract DE-AC05-84OR21400 with the U.S. Department of Energy

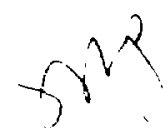


measurement of direct photons. Thermal, directly radiated photons provide us, in principle, with an excellent means to study the QGP since they are essentially uninfluenced by the hadrorization process because of their electroweak interaction. However, in the case of nucleus-nucleus collisions, spectra of direct photons are extremely difficult to deduce from photon measurements due, primarily, to the dominating effect of the combinatorial background arising from the decay of the multitude of neutral pions which are produced in these reactions. In addition, hadronic showers resu'ting mostly from the large number of charged pions contribute in an important way to our systematic errors. The current status of our direct photon emission results is discussed here. One important by-product of our efforts to determine direct photon spectra is a set of distributions of neutral pions which, in themselves, provide us with interesting information on the nature of the compressed and highly excited reaction zone. The transverse momentum $\left(p_{T}\right)$ spectra of these neutral pions are also presented in this paper.

\section{GLOBAL CHARACTERISTICS OF NUCIEUS-NUCLEUS COLLISIONS}

\section{AT CERN SPS ENERGIES}

The three primary sources of data that have led to the elucidation of the global properties of the investigated reactions are energy measurements at zero degrees $\left(E_{Z D C}\right)$, measurements of transverse energy $\left(E_{T}\right)$, and measurements of the multiplicity of charged particles $\left(N_{c}\right)$. It was found that the three quantities $E_{Z D C}, E_{T}$, and $N_{c}$ are strongly correlated with each other. Thus, any one of the experimentally determined quantities can serve as a guide to the impact parameter associated with any given event. As an example, we show in Fig. 1 the correlation between $E_{Z D C}$ and $E_{T}$.

\section{1. $E_{Z D C}$, reaction geometry, and nuclear stopping}

Measurements of the energy remaining at zero degrees (pseudorapidity $\eta \geq 6.0$ ) yield a large amount of important information. $E_{Z D C}$ spectra from $200-\mathrm{A}-\mathrm{GeV}^{32} \mathrm{~S}$ collisions with various target nuclei are shown in Fig. 2. The geometrical features of the various reactions are obvious. For example, when the projectile is larger than the target, a significant value of $E_{Z D C}$ is always observed, due to projectile spectator nucleons that do not intcract with the relatively small target nucleus. On the other hand, when the projectile is much smaller than the target nucleus (e.g., the $S+A u$ case), a relatively large yield is observed at low $E_{Z D C}$ values. The observed peak (near $800 \mathrm{GeV}$ ) is a result of the fact that a relatively broad range of impact parameters leads tn essentially the same situation: the entire projectile interacts primarily with a "tube" of nucleons at (or near) the center of the target nucleus. The fact that the observed peak is located at a finite value of $E_{Z D C}(\sim 800 \mathrm{GeV})$, rather than at $E_{Z D C}=0$, is an indication that at $200 \mathrm{GeV} /$ nucleon, a certain number of participating nucleons, and/or noninteracting projectile nucleons, is always present at beam rapidity as a result of incomplete nuclear stopping.

The above nuclear stopping considerations can be put on a semiquantitative basis. This was first done by Stachel and Braun-Munzinger with reference to our $E_{Z D C}$ data. ${ }^{3}$ From the 
peaks at the low end of the $E_{Z D C}$ spectra, they deduced the number of noninteracting nucleons $\left(n^{n i}\right)$ resulting in an estimate of the stopping fraction $\left(f_{0}\right)$, given by $f_{0}=\left(A_{p}-N_{p}^{n i}\right) / A_{p}$, where $A_{p}$ is the total number of projectile nucleons. The values of $f_{0}$ were found

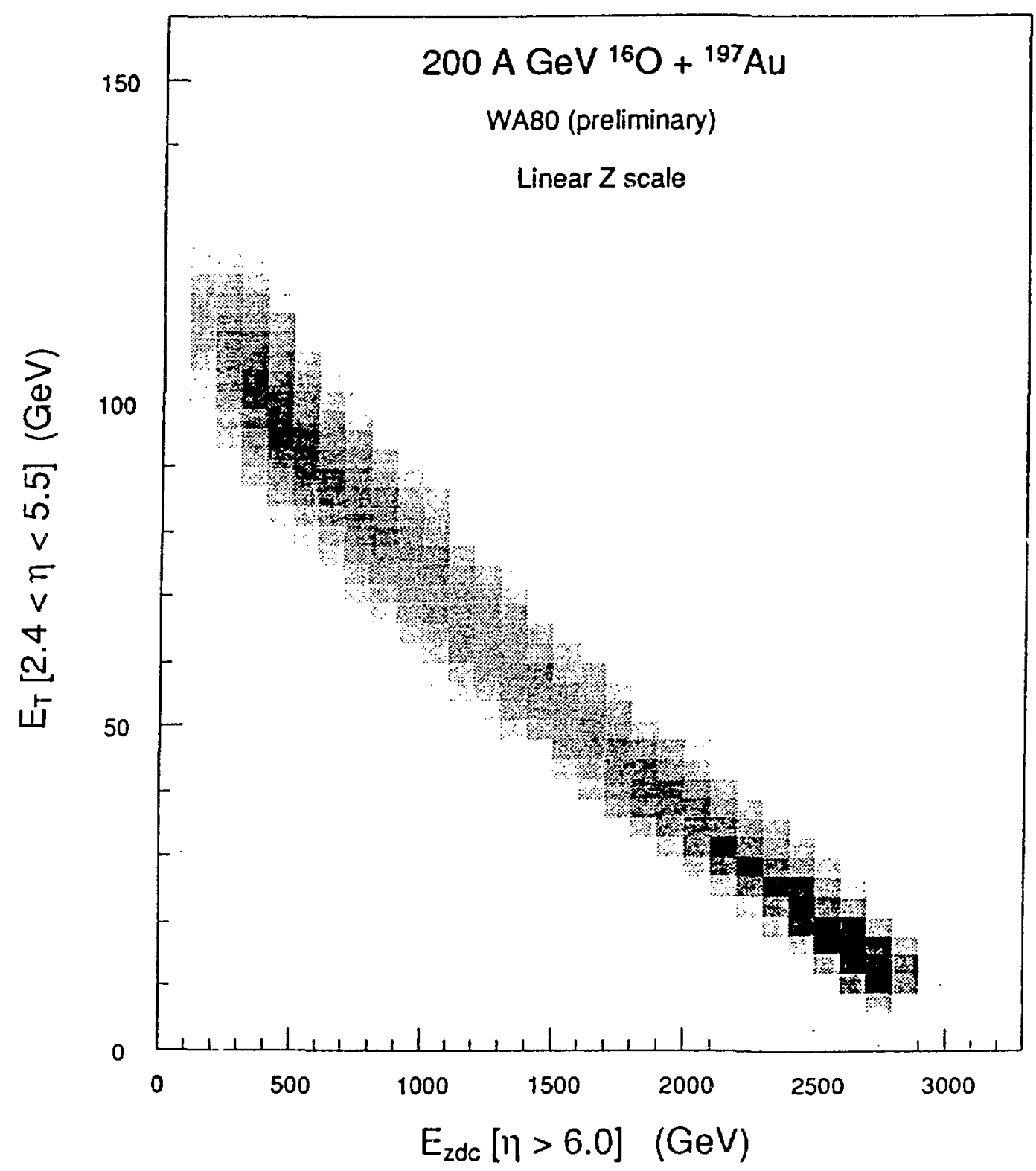

FIGURE 1

Correlation between the transverse energy $E_{T}$ measured in the pseudorapidity range $2.4<\eta<5.5$ and the energy measured at zero degrees in $200-\mathrm{A}-\mathrm{GeV}$ collisions of ${ }^{16} \mathrm{O}$ and ${ }^{19} \mathrm{Au}$. 


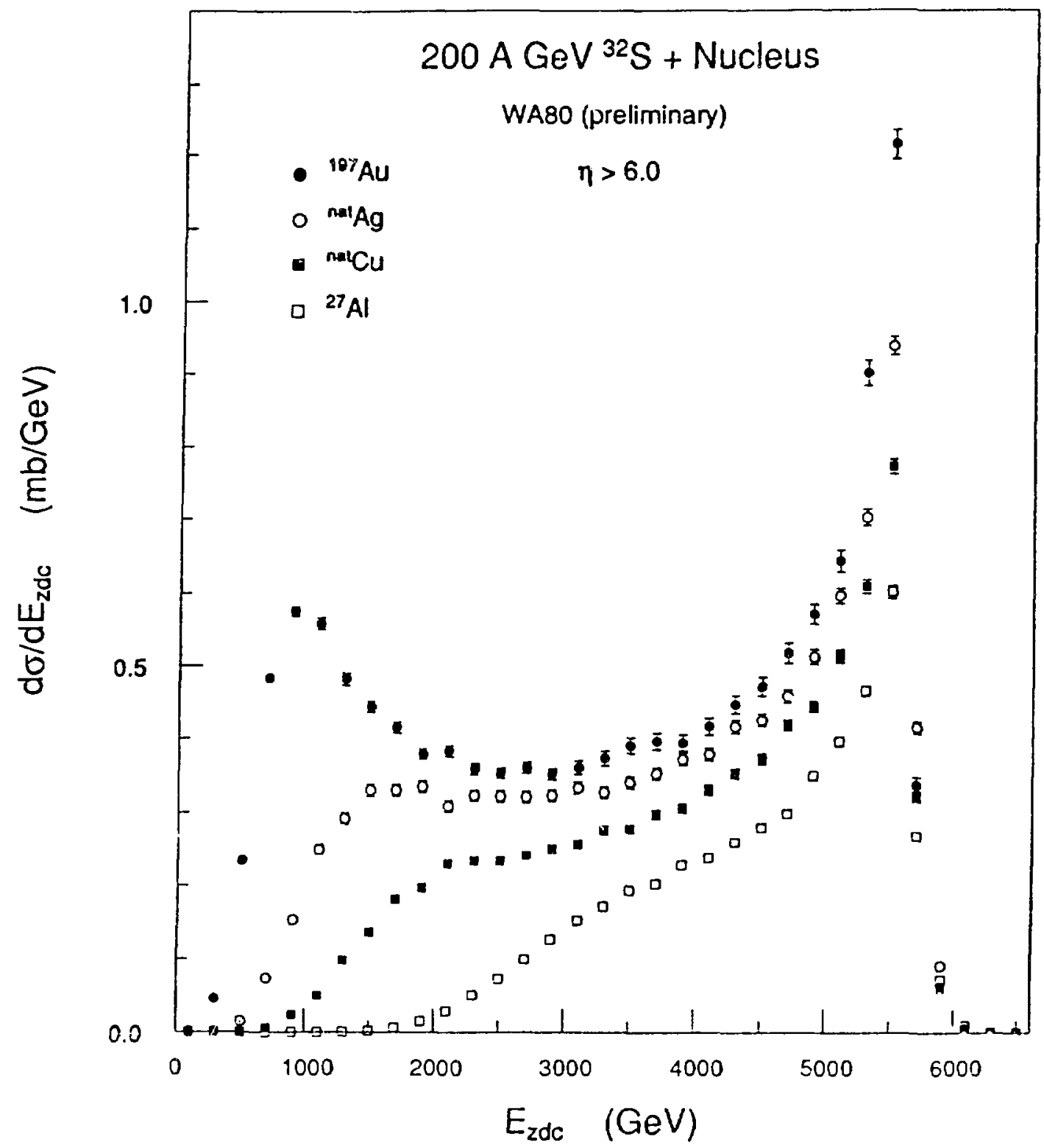

FIGURE 2

Energy distributions measured with the Zero-Degree Calorimeter from reactions of $200-\mathrm{A}-\mathrm{GeV}^{32} \mathrm{~S}$ projectiles with various targets.

to range from 0.88 to 0.98 at $60 \mathrm{GeV} /$ nucleon and from 0.72 to 0.88 at $200 \mathrm{GeV} /$ nucleon. It is safe to conclude that at CERN energies the degree of nuclear stopping is relatively large, but not complete. 
2.2. Transverse energy distributions

In Fig. 3 we show $E_{T}$ distributions measured in the pseudorapidity range $2.4<\eta<5.5$ for $\mathrm{O}+\mathrm{Au}$ and $\mathrm{S}+\mathrm{Au}$ reactions. Both the energy dependence and the target dependence are illustrated. The large increase in $E_{T}$ with bombarding energy has to be reflected in a corresponding increase in the energy density, since, for a given system,

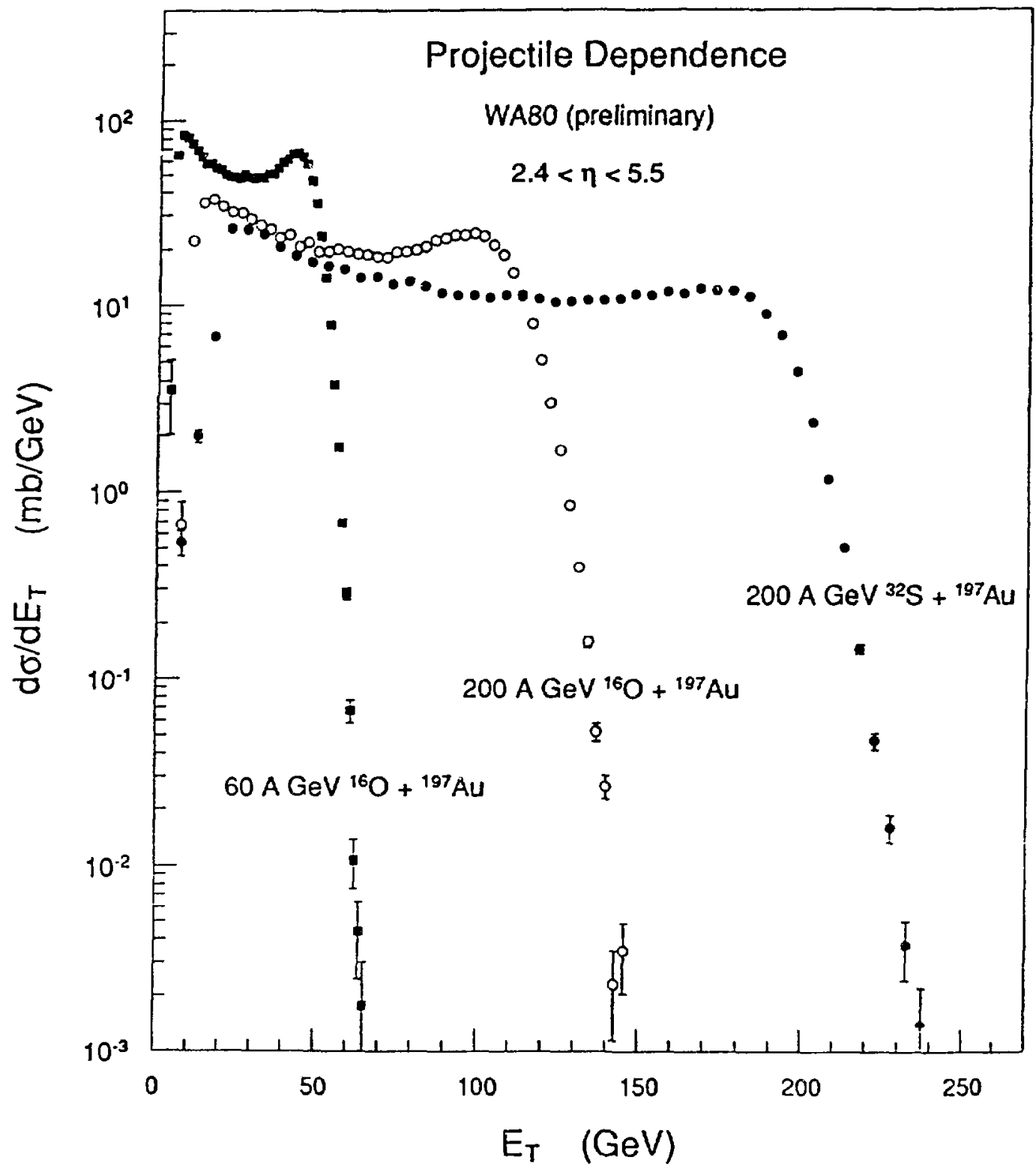

FIGURE 3

Transverse energy distributions from $200-\mathrm{GeV} /$ nucleon reactions of ${ }^{16} \mathrm{O}$ and ${ }^{32} \mathrm{~S}$ with Au and from $60-\mathrm{GeV} /$ nucleon reactions of ${ }^{10} \mathrm{O}$ with $\mathrm{Au}$. 
the associated volume is likely to remain approximately constant. This is not true when different reactions are compared. Thus, the increase of $E_{T}$ in the sulphur case relative to the $O$ case is accompanied by a corresponding increase in the reaction volume, resulting in only a small increase in the estimated energy density.

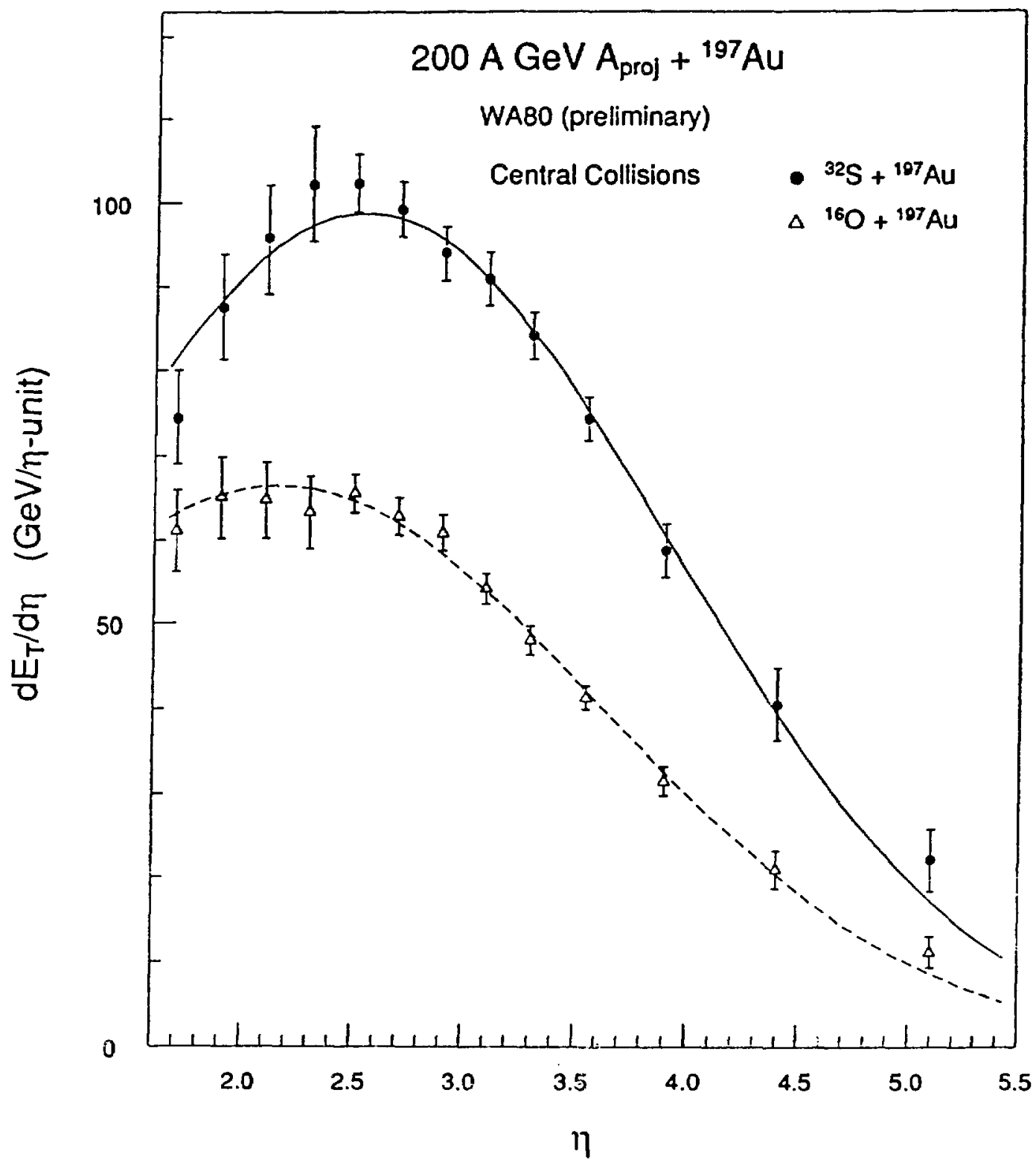
$\mathrm{GeV} /$ nucleon ${ }^{16} \mathrm{O}$ and ${ }^{32} \mathrm{~S}$ with $\mathrm{Au}$ target nuclei. 
A number of estimates have been made tha: predict the scaling of $E_{T}$ as a function of the projectile size. They are usually based on estimates of the number of participating nucleons in the clean-cut "tube" geometry. Experimental results of the various collaborations differ from each other due to the different $\eta$ coverages involved. The situation can be elucidated with reference to Fig. 4, which gives the pseudorapidity distributions $\left(d E_{T} / d \eta\right)$ from central collisions of $\mathrm{O}+\mathrm{Au}$ and $\mathrm{S}+\mathrm{Au}$ at $200 \mathrm{GeV} /$ nucleon. It can be seen that the scaling from one system to the other depends on the region of $\eta$ that one is considering. Thus, for example, at very high $\eta$ values, where effects of the projectile dominate, the S+Au distribution lies almost a factor of two above the $\mathrm{O}+\mathrm{Au}$ distribution. At midrapidity (somewhat below $\eta=3$ ), the scaling between the two curves is close to $A_{p}^{2 / 3}$, expected on the basis of the "tube" geometry. Finally, at the lowest $\eta$ values, where target effects dominate, the scaling is well below the $A_{p}^{2 / 3}$ value.

A set of $d E_{T} / d_{i j}$ distributions for $O$-induced reactions at $60 \mathrm{GeV} /$ nucleon are shown in Fig. 5. Two observations are relevant. First, the peaks of the distributions are seen to shift to lower rapidities with increasing mass of the target nucleus, indicating more slowly moving effective c.m. systems. Second, experiments with only limited coverage in the forward rapidity region (e.g., $\eta \gtrsim 3$ ) would observe very similar $d \sigma / d E_{T}$ distributions for all systems (except $\mathrm{O}+\mathrm{C}$ ) from which the erroneous conclusion could be drawn that the degree of stopping is the same (and complete) in all cases. In addition, although not shown, the widths of the $d E_{T} / d \eta$ distributions are significantly broader than those expected to result from an isotropic fireball, and they are found to decrease with increasing centrality. This is accompanied by an increase in the number of contributing sources (participating nucleons and/or number of collisions).

\subsection{Energy densities}

The quantity crucial to the probability of QGP formation is the thermalized energy density attained during the collision. It is possible to estimate energy densities, $\epsilon$, from the $E_{T}$ measurements and to extrapolate these findings to systems, such as $\mathrm{Pb}+\mathrm{Pb}$, that have not been investigated. The most widely used method by which energy-density estimates are made is due to Bjorken. Strictly speaking, this method applies only to the extreme relativistic limit, and may not be appropriate at CERN energies. Furthermore, the Bjorken approach involves a formation time $r$ which is not known and which is usually estimated to be $1 \mathrm{fm} / \mathrm{c}$. This method involves boost invariance and allows us to obtain differential energy-density estimates valid for each local rest frame. While we have estimated energy densities under various alternative assumptions which do not rely on the existence of a plateau at midrapidity, ${ }^{5}$ the Bjorken approach provides us with a means of making system-to-system comparisons that is not surpassed at the present time. Using our experimental, preliminary $d E_{T} / d \eta$ distributions, we found the standard Bjorken volume-averaged energy densities to vary from about $1.0 \mathrm{GeV} / \mathrm{fm}^{3}$ for ${ }^{16} \mathrm{O}+{ }^{12} \mathrm{C}$ to about $2.5 \mathrm{GeV} / \mathrm{fm}^{3}$ for ${ }^{16} \mathrm{O}+{ }^{197} \mathrm{Au}$. In addition, by 


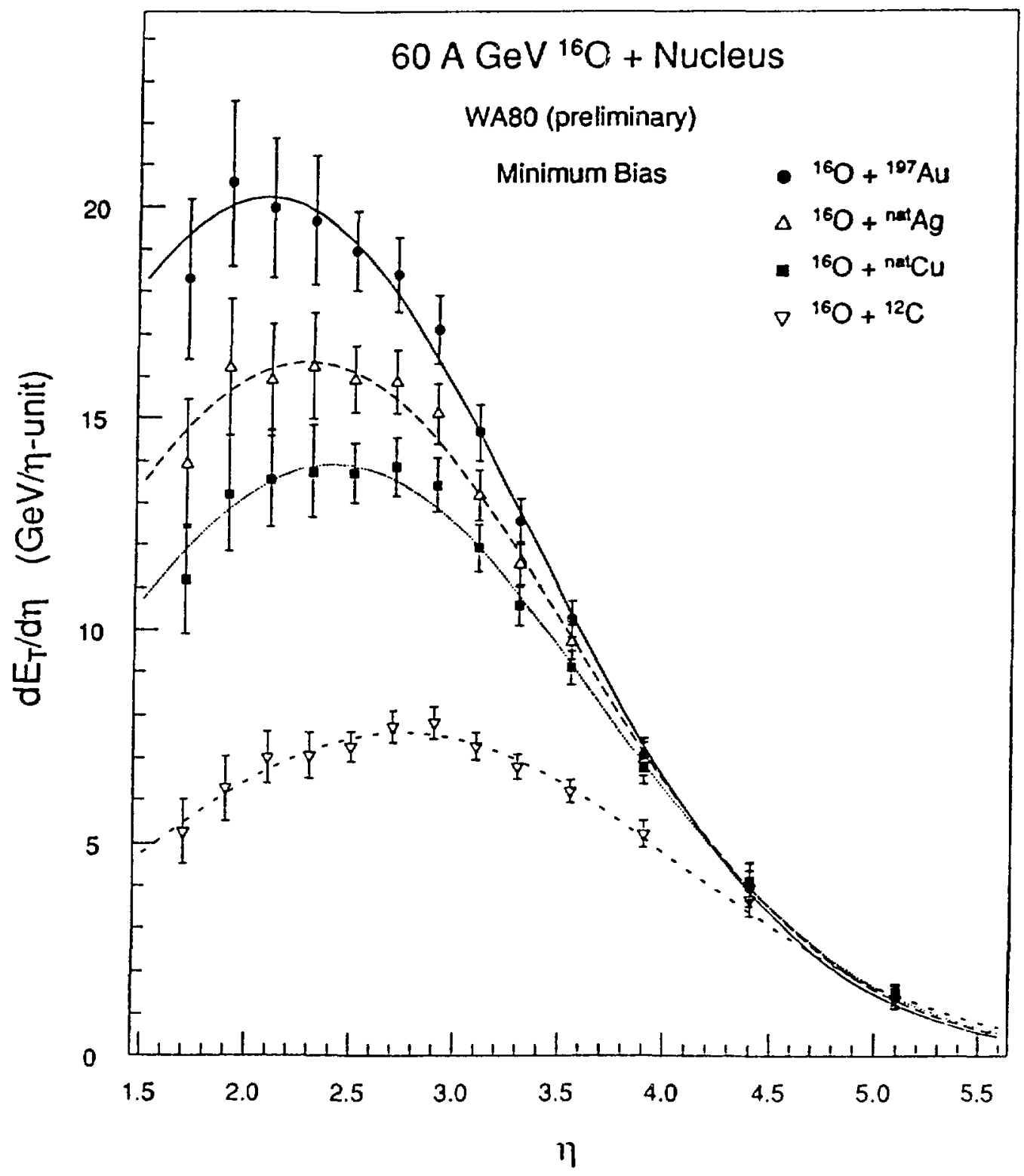

FIGURE 5

Transverse energy pseudorapidity distributions from minimum-bias reactions of 60 $\mathrm{GeV} /$ nucleon ${ }^{16} \mathrm{O}$ with various targets.

making use of the $E_{T}$ /participant values described above, we estimate that the volumeaveraged energy density sttained in a central $\mathrm{Pb}+\mathrm{Pb}$ collision will be about2.7 $\mathrm{GeV} / \mathrm{fm}^{3}$. The two points to note are that the above values of energy density $\left(2-3 \mathrm{GeV} / \mathrm{fm}^{3}\right)$ are within the range of values estimated to be required for $\mathrm{QGP}$ formation, and that $\mathrm{Pb}+\mathrm{Pb}$ collisions do not involve much higher volume-averaged energy densities than $\mathrm{G}+\mathrm{Pb}$ collisions. 
An important finding of the WA80 experiment is that $d E_{T} /\left.d \eta\right|_{\max }$ per participant is about $1 \mathrm{GeV}$ for reactions at $200 \mathrm{GeV} /$ nucleon. This result makes it possible to estimate energy densities as a function of the radial profile of the colliding system. This result was first discussed in Ref. 5. It was brought out that in the central core of a head-on S+Au collision at $200 \mathrm{GeV} /$ nucleon, the energy density is expected to reach $3 \mathrm{GeV} / \mathrm{fm}^{3}$, while in a head-on Au+Au collision the central energy density may reach a value as high as $4 \mathrm{GeV} / \mathrm{fm}^{3}$. Since, in addition to the higher core energy densities, large colliding nuclei may involve a larger volume of QGP formation, the development of heavy beams at the CERN SPS should be pursued. It should be stressed, however, that the most effective way of increasing the energy density is by increasing the collision energy; and it is possible that sufficiently high energy densities for QGP formation may not be attained until the Relativistic Heavy-Ion Collider is built at BNL.

\section{PHOTON MEASUREMENTS}

Photon measurements have been carried out by means of a single-arm lead-glass photon detector ${ }^{6}$ (SAPHIR) of modest acceptance $\left(\Delta \phi=2 \pi / 3\right.$ and $13.7^{\circ} \leq \theta \leq 25.9^{\circ}$ ) and of limited resolution $(\sigma / E=0.4 \%+6 \% / \sqrt{E})$. We present here our results on the spectra of direct photons as well ar transverse momentum spectra of neutral pions.

\subsection{Direct photons}

As was stated in the introduction, the primary purpose of SAPHIR is to measure the spectra of direct photons which may be emitted from the QGP. The procedure used to deduce these spectra relies on our ability to account for all photons impinging on the surface of SAPHIR that result from known hadronic decays. The difficulties associated with this task can be appreciated when we consider the uncertainties associated with SAPHIR's efficiency (in a high-multiplicity environment), its limited acceptance (making certain invariant-mass reconstructions impossible), and the unknown transverse momentum distributions of the other hadronic decays that contribute to the background $\left(\eta, \omega\right.$, and $\left.\eta^{\prime}\right)$ in addition to the dominant neutral pions. ${ }^{7,8}$

In mid-1988, we reported preliminary results on direct photon emission from reactions induced with ${ }^{16} \mathrm{O}$ projectiles at $200 \mathrm{GeV} /$ nucleon. ${ }^{9}$ Subsequent work on the evaluation of SAPHIR efficiency and acceptance, however, has led to a revision of our preliminary findings. ${ }^{10}$ The revised results are shown in Fig. 6 in which the $\gamma / \pi^{0}$ ratio is shown for central and peripheral ${ }^{16} \mathrm{O}+\mathrm{Au}$ reactions at $200 \mathrm{GeV} /$ nucleon as a function of $p_{T}$. The Monte Carlo calculations depicted in the figure give the contribution of all decay photons. The Monte Carlo procedure and its assumptions are described elsewhere. ${ }^{8}$ Within the limits of uncertainties, all of the observed photons are accounted for by known hadronic decays. Given all sources of uncertainties, we set un upper limit of about $15 \%$ for the contribution of direct photons to the $\gamma / \pi^{0}$ ratio in our $p_{r}$ range. With better statistics, it should be possible to set a limit at the $5 \%$ level. 


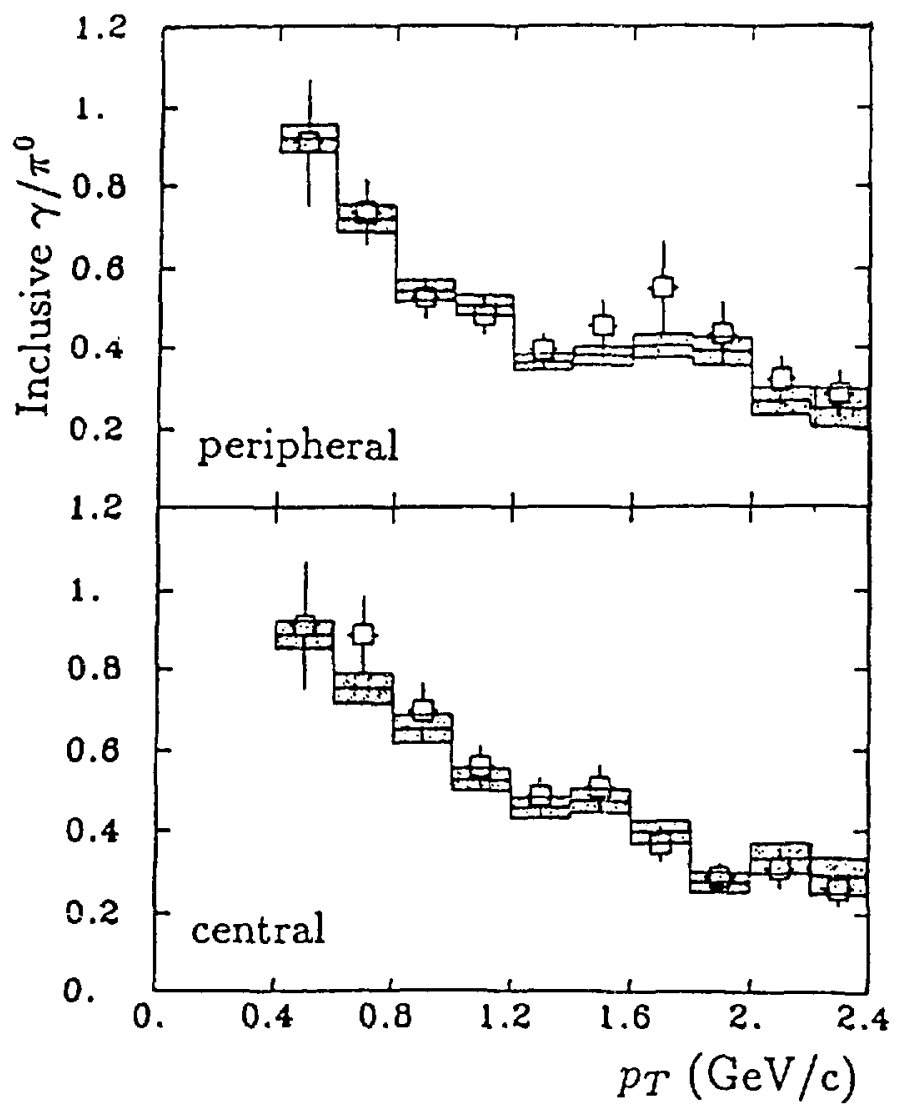

FIGURE 6

Ratio of inclusive photon cross section to $\pi^{0}$ cross section as a function of transverse momentum for central and peripheral ${ }^{16} \mathrm{O}+\mathrm{Au}$ reactions at 200 $\mathrm{GeV} /$ nucleon. The squares indicate data, and the shaded histograms are Monte Carlo results representin 5 hadronic decays. The difference between the data and the histograms can be atiributed to the contribution of direct photons.

Photon measurements at very low $p_{T}$ are most interesting since the direct photon radiation from possible QGP formation is expected to yield photons with $p_{T}$ dominantly in the region of up to several times the plasma temperature of about $200 \mathrm{MeV}$. However, the extraction of the $\pi^{0}$ and $\eta$ cross sections at low $p_{T}$ is most difficult, due to the necessary requirement of a large acceptance in relative photon angles $\Delta \phi$, and hence, a large acceptance detector is required. Also, the background from the combination of false photon pairs increases for decreasing $p_{T}$, necessitating a detector with very good mass resolution for the $\pi^{0}$ and $\eta$. In the next period of WA80 running with sulphur beams, scheduled for the summer of 1990, the present coverage with lead-glass detectors will be tripled, leading to a significantly improved $\pi^{0}$ and $\eta$ acceptance at low $p_{T}$. In addition, in 1991 an array of 
several hundred BGO modules will be used to determine whether the expected improvement of a factor of 4 to 5 in mass resolution can be obtained in our high-multiplicity environment. Such an improvement in mass resolution is necessary for a possible photon experiment with $\mathrm{Pb}$ beams at CERN in order to cope with the expected increase in the combinatcrial background resulting from the higher photon multiplicity.

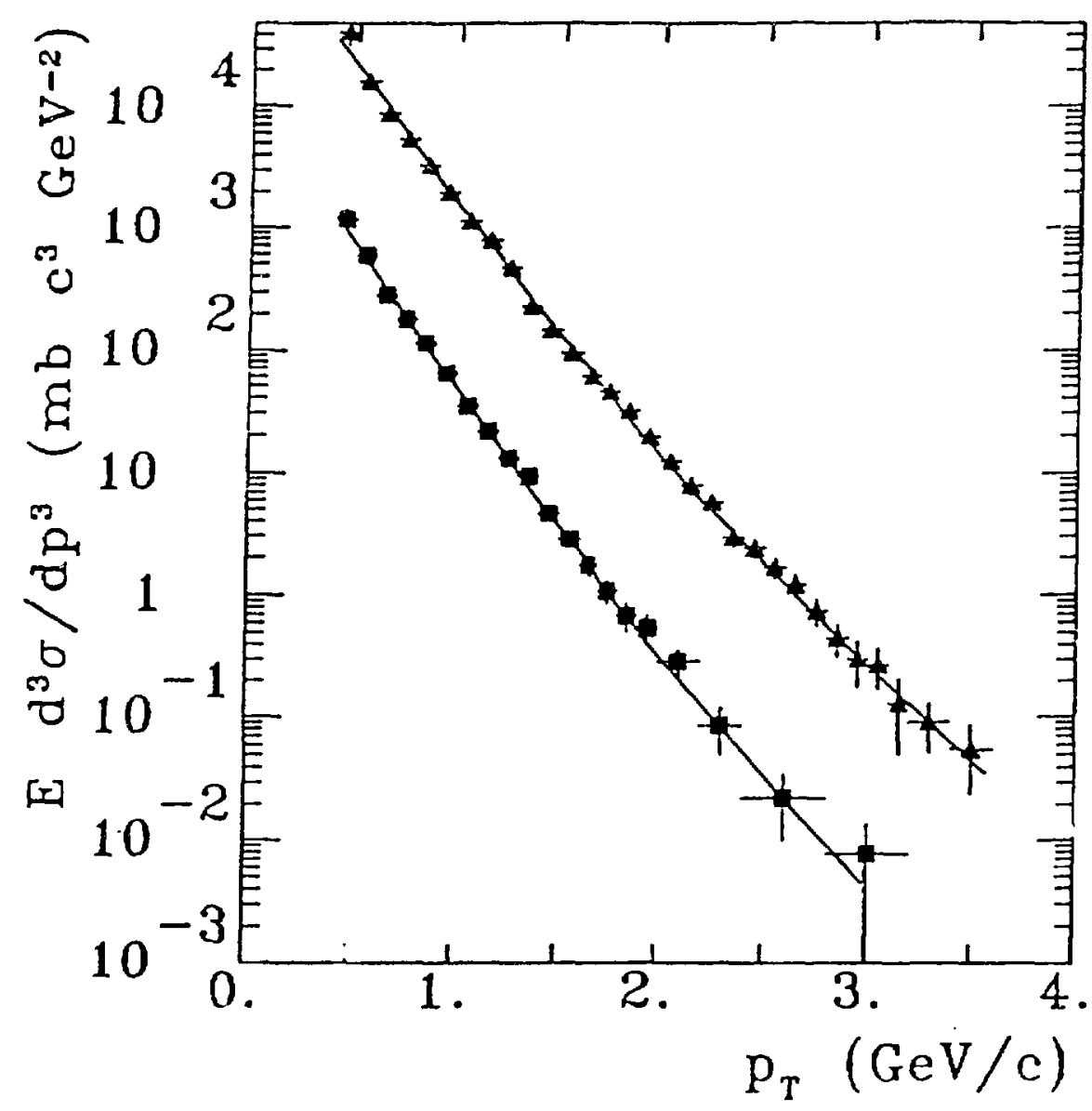

FIGURE 7

Invariant $\pi^{0}$ cross sections from minimum-bias collisions of $p$ (squares) and ${ }^{16} \mathrm{O}$ (triangles) with Au target nuclei at $200 \mathrm{GeV} /$ nucleon measured in the pseudorapidity range $1.5 \leq \eta \leq 2.1$. The lines represent fits to the data of Eq. (1) and parameters of Table 1.

\subsection{Transverse momentum distributions of neutral pions}

The investigation of the $\pi^{0}$ spectra presented here covers a $p_{T}$ region up to $3.6 \mathrm{GeV} / \mathrm{c}$ and involves the selection of data according to collision centrality. The results cover the full data set of the $1986{ }^{16} \mathrm{O}+\mathrm{Au}$ run and extend the subset of data published in Ref. 11 . They are described together with data analysis and efficiency and acceptance corrections in a forthcoming publication. ${ }^{7}$ Only selected highlights are presented here. In Fig. 7 invariant 
$\pi^{0}$ cross sections are shown for minimum-bias collisions of protons and ${ }^{16} \mathrm{O}$ projectiles with Au nuclei at $200 \mathrm{GeV} /$ nucleon, and in Fig. $8 \pi^{\circ}$ cross sections are shown for central and peripheral $200-\mathrm{A}-\mathrm{GeV}{ }^{16} \mathrm{O}+\mathrm{Au}$ events. Attempts to fit the spectra with a combination of two thermal distributions did not give reliable results since the two extracted temperatures

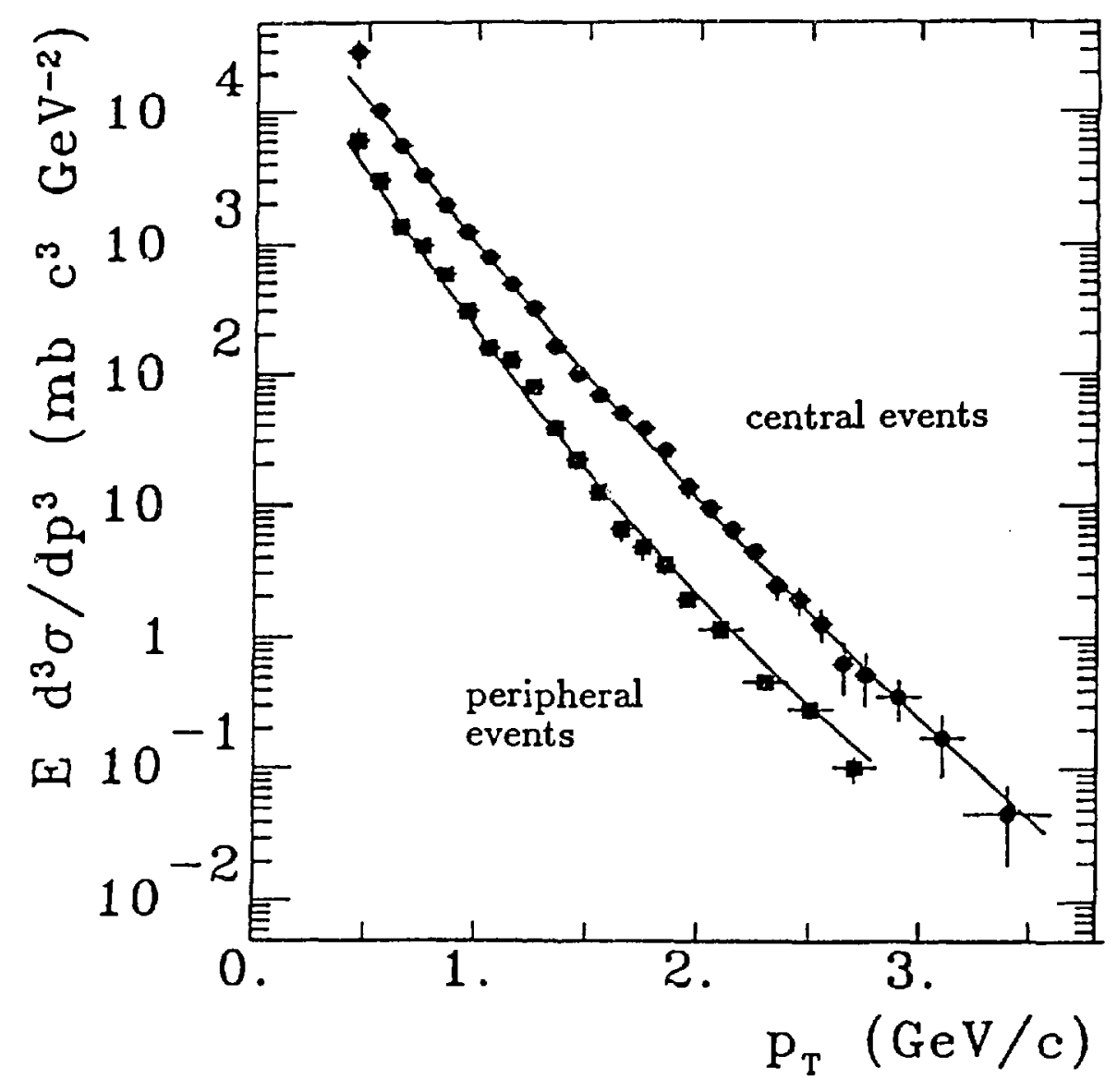

FIGURE 8

Invariant $\pi^{0}$ cross sections from central (circles) and peripheral (squares) collisions of ${ }^{16} \mathrm{O}$ projectiles with an $\mathrm{Au}$ target at $200 \mathrm{~A} \mathrm{GeV}$ measured in the pseudorapidity range $1.5 \leq \eta \leq 2.1$. The lines represent fits to the data with Eq. (1) and parameters of Table 1.

turned out to be sensitive to both the relative normalization and to statistical fluctuations. Following Hagedorn ${ }^{12}$ the spectra were parametrized by:

$$
E \frac{d^{3} \sigma}{d p^{3}}= \begin{cases}A \exp \left(-m_{T} / T\right) & \text { for } p_{T} \leq 0.8 \mathrm{GeV} / \mathrm{c} \\ C\left(\frac{p_{0}}{p_{T}+p_{0}}\right)^{n} & \text { for } p_{T} \geq 0.8 \mathrm{GeV} / \mathrm{c}\end{cases}
$$


where $A, T, C, p_{0}$, and $n$ are parameters and $m_{T}$ is the tran verse mass, $m_{T}=\left(p_{T}^{2}+\right.$ $\left.\mathrm{m}^{2}\right)^{1 / 2}$. The first expression is an approximation to a thermal distribution, while the second expression is empirical and based on QCD considerations. Two parameters are eliminated by the requirem nt that the values and slopes of the two expressions match at $p_{T}=0.8 \mathrm{GeV} / \mathrm{c}$. The fit values of the remaining three parameters, $A, T$, and $n$, are given in Table $l$ for the data of Figs. 7 and 8. The fitted curves are also shown in the figures. The two points to be noted are that the value of the parameter $T$ remains constant (within experimental errors) for all data sets, and that the parameter $n$ varies from one data set to another, indicating differences in the spectra at $p_{T}$ values above $0.8 \mathrm{GeV} / \mathrm{c}$. The differences in the high $p_{T}$ region become more apparent when comparisons are made to data from $p+p$ collisions and when ratios of spectra are plotted as a function of $p_{T}$. A marked change of slope at higher values of transverse momentum is seen in the $p+A u$ case anc has previously been ascribed to the onset of hard QCD scattering. ${ }^{13}$ This effect is also found in our peripheral-collision ${ }^{16} \mathrm{O}$ data, but not in results from central collisions where, presumably, the effect is obscured by nuclear effects.

Table 1. Parameters obtained from fitting Eq. (1) to experimental data of Fig. 1 and to minimum-bias data from $\mathrm{p}+\mathrm{Au}$ and ${ }^{16} \mathrm{O}+\mathrm{Au}$ reactions at $200 \mathrm{GeV} /$ nucleon.

\begin{tabular}{lccc}
\hline System & $A\left(\mathrm{mb} \mathrm{c}^{3} \mathrm{GeV}^{-2}\right)$ & $T(\mathrm{MeV} / \mathrm{c})$ & $n$ \\
\hline \hline $200-\mathrm{GeV} \mathrm{p}+\mathrm{Au}$ & $(1.3 \pm 0.5) 10^{4}$ & $180 \pm 16$ & $37.6 \pm 3.9$ \\
\hline $\begin{array}{l}200-\mathrm{A}-\mathrm{GeV} \text { O+Au } \\
\text { minimum-bias }\end{array}$ & $(3.6 \pm 1.3) 10^{5}$ & $184 \pm 16$ & $25.9 \pm 2.4$ \\
peripheral & $(6.6 \pm 2.9) 10^{4}$ & $174 \pm 18$ & $16.8 \pm 1.9$ \\
central & $(1.7 \pm 0.8) 10^{5}$ & $194 \pm 22$ & $26.0 \pm 3.1$ \\
\hline
\end{tabular}

\section{REFERENCES}

1) R. Albrecht et al., Phys. Lett. B 199, 297 (Dec. 1987).

2) S. P. Sorensen, private communication regarding preliminary WA80 data.

3) J. Stachel and P. Braun-Munzinger, Phys. Lett B 216, 1 (1989).

4) J. D. Bjorken, Phys. Rev. D 27, 140 (1983).

5) S. P. Sorensen et al., in Proceedings, 6th International Conference on Ultrarelativistic Nucleus-Nucleus Collisions, Schloss Nordkirchen, F.R.G., August 24-28, 1987, Z. Phys. C 38, 3 (1988); T. C. Awes et al., pp. 485-90 in Proceedings, XXIV Recontres de Moriond, Les Arcs, Savoie, France, March 13-19, 1989, Les Editions Frontieres, 1989.

6) H. Baumeister et al., submitted to Niclear Instruments and Methods in Physics Research. 
7) R. Albrecht et al., "Transverse Momentum Distributions of Neutral Pions from Central and Peripheral ${ }^{16} \mathrm{O}+\mathrm{Au}$ Collisions at $200 \mathrm{~A} \mathrm{GeV}$," submitted to Zeitschrift fuer Physik $A$.

8) L. Dragon, "Produktion neutraler Pioinen und direkter Photonen in ultrarelativistischen Schwerionenreaktion," Dissertation, Institut fuer Kernphysik, WWU Muenster, 1989.

9) F. Santo et al., Proceedings, Seventh International Conference on Ultrarelativistic Nucleus-Nucleus Collisions, Lenox, Massachusetts, September 26-30, 1988; Nucl. Phys. A498, 391c (July 1989).

1ii) L. Dragon, in Proceedings, International Conference on Ultrarelativistic NucleusNucleus Collisions, Menton, France, May 7-11, 1990, to be published.

11) R. Albrecht et al., Phys. Lett. B 201, 390 (Feb. 1988).

12) R. Hagedorn, Rivista del Nuovo Cimento 6, 1 (1983).

13) M. A. Faessler, Phys. Rep. 115, 1 (1984).

\section{DISCLAIMER}

This report was prepared as an account of work sponsored by an agency of the United States Government. Neither the United States Government nor any agency thereof, nor any of their employees, makes any warranty, express or implied, or assumes any legal liability or responsibility for the accuracy, completeness, or usefulness of any information, apparatus, product, or process disclosed, or represents that its use would not infringe privately owned rights. Reference herein to any specific commercial product, process, or service by trade name, trademark, manufacturer, or otherwise does not necessarily constitute or imply its endorsement, recommendation, or favoring by the United States Government or any agency thereof. The views and opinions of authors expressed herein do not necessarily state or reflect those of the United States Government or any agency thereof. 